\title{
Sobreposição da mutação ganho-de-função* do gen SETBP1 na Síndrome de Schinzel-Giedion e em doenças hematológicas malignas
}

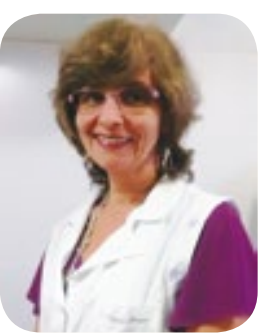

Viviane Borges Ferreira

Publicado no PLOS Genetics. Volume 3, Issue 1, February 2017. Com o mesmo título. Autores: Rocio Acuna-Hidalgo, Pelagia Deriziotis, MarloesSteehouwer,
Christian Gilissen, Sarah A. Graham, Sipko van Dam, Julie Hoover-Fong, Viviane Ferreira, et. al.

RESUMO

A Síndrome de Schinzel-Giedion (SGS) é um raro distúrbio do desenvolvimento, caracterizado por múltiplas malformações, alterações neurológicas severas e elevação do risco de ocorrência de neoplasias malignas. SGS é causada por mutação de novo no hotspo (região do material genético mais propensa a mutação) do braço curto do cromossomo 12 , no exon ${ }^{4}$, no gen SETBP1.

Mutações nesse hotspot interrompem a degradação e regulação proteica, promovendo o acúmulo da proteína SETBP1.

A sobreposicão de mutacões no hotspot do gen da A són proteina SETBP tem sido observada, de maneira re-

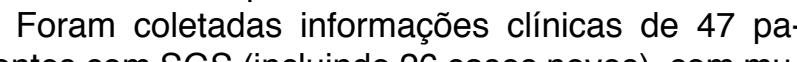
cientes com SGS (inclundo 26 casos novos), com mutaçáo de celulas (incluinativas de SETBP1 e em quatro individuos com fonotipo moderado causado por muta-

Mutaços de ganho de funçăo são aquelas que causam a superexpressão de um produlo gênico (hiomorfismo).

\section{INTRODUCC̃̃o}

Síndrome de Schizel-Giedion (SGS; OMIM 269150) é uma rara doença do desenvolvimento, caracterizada por múltiplas malformacões, incluindo hipoplasia da porção média da face, defeitos cardíacos, hidronefrose e anormalidades esqueléticas. Essa sindrome, re作 doença dominante, de causa genética, foi identificada pelo sequenciamento do exoma. Em 12 de 13 indivíduos com essa doença, foram identificadas mutações de novo de células germinativas, em grupamentos de hotspot de SETBP1, de 12 pares de bases que codificam os resíduos 868 para 871 na proteína SETBP1.

Curiosamente, pouco depois, também foram identificadas mutações de novo de SETBP1, em células germinativas, como causa de SGS, sobrepondo-se a mutações somáticas em SETBP1, em pacientes com eucemia mieloide.

Esse duplo papel no câncer e no desenvolvimento não é único para o gen SETBP1. Outras mutações que provocam defeitos no desenvolvimento também provocar câncer.

Esses achados não são inesperados. Várias crianças com câncer podem apresentar defeitos no desenvolvimento e vice-versa. Parece haver similaridade molecular entre anormalidades na embriogênese e desenvolvimento de câncer.

A função precisa da proteína SETBP1 ainda é desconhecida. O que se sabe é que a mutação de SETBP1 nas células germinativas provoca estabilização dessa proteina, dificultando sua degradação e provo-

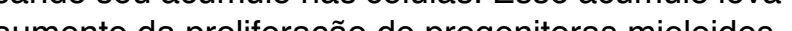

\section{RESULTADOS}

\section{Achados clínicos na SGS}

Dismorfismos faciais:

Todos os indivíduos com SGS têm características faciais facilmente reconhecíveis (Fig 1C).

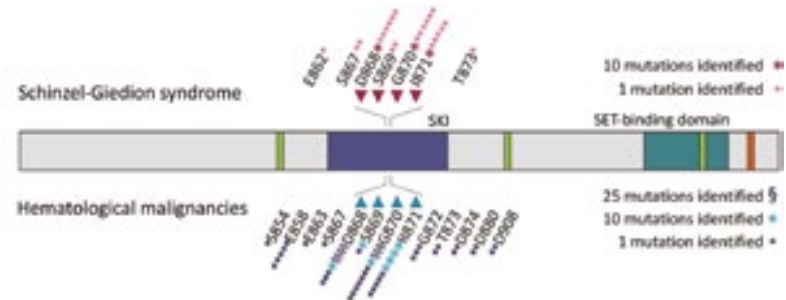

B
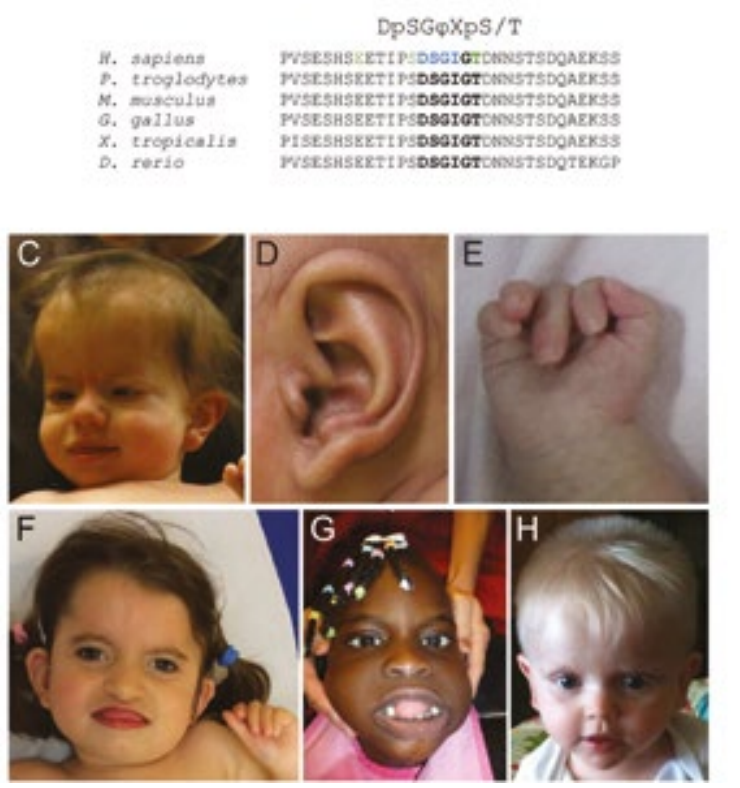

Figura 1. Características genéticas e clínicas de indivíduos com mutações SETBP1 da linha germinal e Síndrome de Schinzel-Giedion. A. Representação esquemática da proteína SETBP1, indicando alterações encontradas na SGS e em malignidades hematológicas. B. Alinhamento de seqüência da região contendo a degradação de SETBP1. C. Características faciais distintivas encontradas na SGS clássica. D. Orelha típica em forma de ponto de interrogação. E. Postura de mão característica com aperto de dedos. F. Características faciais e os dedos apertados. G. Características faciais. H. Características faciais.

Fronte ampla, predomínio da distância ântero-posterior sobre a bitemporal, olcos proeminentes, hipertelorismo, terço medio da face retraido e nariz pequeno. Alguns casos têm implantação anormal das orelhas. As orelhas são pequenas e rodadas posteriormente, com angulação anterior do lobo da orelha.

Alguns indivíduos não apresentam essas caracteristicas ao nascimento, mas ate os 18 meses são bem evidentes. Nesses casos, alguns achados podem ajudar, como implantação anormal das orelhas. Classicamente, as orelhas dos indivíduos com SGS têm implantação baixa, com rotação posterior e angulação anterior dos lobos dando a impressão de ponto de interrogação. Em individuos que não têm os lobos típicos a maioria tem orelhas em formato de hélice ou proeminentes anti-helices. Cerca de metade dos pacientes apresentavam boca grande, lábio inferior evertido e lingua protusa. A maioria apresentou micrognatia, filtro sulcado. Dois terços dos pacientes apresentam hipertricose. Oito de 33 apresentave 33 tinham pescoço curto.

As alterações esqueléticas foram vistas em $71 \%$ dos pacientes, incluindo esclerose da base do crânio, com sincondrose occipital, afastamento das costelas, ramo púbico pequeno, sínfise púbica ampla, hipoplasia das falanges distais das mãos e dos pés. Polidactilia ocorreu em $11 \%$ dos casos. Estudos fotográficos retrospectivos de pacientes com SGS mostraram uma postura típica de dedos em garra.

Características neurológicas: microcefalia é observada em cerca de dois terços dos indivíduos com SGS. O perímetro cefálico sempre foi abaixo do percentil 50 frequentemente próximo ao percentil 10. Atraso severo do desenvolvimento ocorre nos indivíduos com SGS e $95 \%$ apresentam epilepsia. Quase todos apresentam crises extremamente refratárias ao tratamento com medicação ou dieta cetogênica. Muitos pacientes apresentam deficiência visual ou auditiva, de origem cerebral. Espasticidade foi observada em 17 de $20 \mathrm{ca}$ sos. Foram observadas malformações estruturais do cérebro, nos pacientes com SGS. A anomalia mais comum foi hipoplasia ou aplasia do corpo caloso. Atrofia cortical em 18 de 33 pacientes, anomalias ventriculares em 26 de 42 pacientes, anomalias nos giros, atraso na mielinização e cistos de plexo coroide.

Anomalias congentitas adicionais

Indivíduos com SGS apresentam hidronefrose, que ja e observada nos exames pré-natais. Dois pacientes de nossa serie já tiveram esse diagnostico na ultrassonografia com 20 semanas de gestação. Foram observadas outras alteraçōes renais, como ureteres anormais, cistos cálculos renais. Quase todos os pacientes apresentaram anomalias genitais, que incluem hipospádia, hipodesenvolvimento da genitália e distopia anal.

Metade dos pacientes apresentaram malformações tras anomalias incluem forame oval patente, permanência do ducto arterioso e hipertrofia cardíaca.

Alguns pacientes apresentaram alterações de órgãos internos como hipoplasia da cauda do pâncreas ou hepatoesplenomegalia. 19 em 27 apresentavam alacrimia. 8 
em 15 apresentavam hérnia inguinal. $11 \mathrm{em} 17$ apresenaram pés equinovarum

Disfagia e dispneia são os maiores problemas aprentados pelos pacientes com SGS.

Esses sintomas são causados por uma combinação de fatores como anomalias no aparato respiratório: estenose de coanas, traqueobronquiomalácea, hipoplasia pulmonar, dificuldade para manejar com a secreção respiratória (causada pela micrognatia, hipertrofia gengival e excessiva produção de muco) e alta suscetibilidade a A

A hipertrofia gengival pode ser causada pelo uso de anticonvulsivantes, mas um recém-nascido mostrou esse sinal clínico com 4 dias de vida.

Os pacientes mais afetados não passam da primeira infância. Pneumonia é a maior causa de morte nos pacientes com SGS. Outras causas são as malformações cardíacas, tumores, hipoplasia pulmonar, convulsões intratáveis e parada cardíaca súbita.

Seis indivíduos desenvolveram tumores sólidos, predominantemente de origem neuroepitelial, na região lombo-sacral. Um indivíduo desenvolveu leucemia mieloide juvenil. Embora a maioria dos indivíduos faleçam na infância, 5 dos 12 pacientes com a substituição proteica do resíduo G870 viveram por 5 anos ou mais (de 5 a 15 anos).

ndivíduos com mutação extra-degron*

Um degron é uma porção de uma proteína que é importante na regulação das taxas de degradação de proteínas.

Indivíduos com mutação na linhagem germinativa SETBP1, extra-degron, apresentam as características faciais, anomalias genitais e convulsões, mas não mostram hidronefrose.

Um indivíduo com mutação no resíduo E862 não mostrou as características faciais, nem convulsões, nem hidronefrose. No entanto, apresentava disfagia, necessitando de gastrostomia, deficiência visual severa por distrofia da retina, cistos renais bilaterais e severa espasticidade. Ela apresentava microcefalia, prognatismo, pés pequenos e artelhos pequenos

Aos 5 anos, apresentava deficiência mental severa, não andava, nem falava.

O indivíduo com mutação no resíduo T873 apresentava atraso no desenvolvimento, caracteristicas de autismo, diplegia espástica e alterações dismórficas moderadas. Não apresentava anomalias congênitas, comumente encontradas na SGS e, a despeito do atraso do desenvolvimento, seu desenvolvimento inicial é maior, comparado com os individuos que têm mutação do SETBP1 com degradação. Apresentou algumas aquisições: sorriu com 8 semanas, emitiu sons articulados aos 14 meses, sentou sem apoio aos 22 meses e, aos 2 anos rastejava, andava com apoio e comia sozinho. Entretanto, entrou em fase de regressão, com perda das aquisções e autoagressão. Aos 4 anos, tinha comportamento le um bebê de 8 meses.

Sobreposição da mutação no gen da proteína SETBP1 na SGS e nas doenças malignas mieloides.

Sobreposição da mutação de novo no gen da proteína SETBP1 tem sido identificada em células germinativas na SGS e em celulas somálicas nas doenças malignas mieloides. Foram comparadas as mutações em células germinativas e em células somáticas.

48 mutações de novo em células germinativas foram servadas nos indivíduos com SGS.

Foram descritos em literatura 245 indivíduos com mutação em ceelulas somaticas no degron de SETBP1 associada a diferentes doenças mieloproliferativas. A sobreposição ocorre em ambas as condiçoes, mas a distribuição da mulação não e a mesma. Um maior número de mutações afetam resíu 1871 nos casos de SGS, com

\section{Aumento na gênese de tumores em indivíduos}

com mutaçoes no resíduo D868.

Sete indivíduos com SGS apresentaram malignidade 5 em pacientes com mutação no resíduo D868 (4 tumores de origem neuroectodérmica e 1 com leucemia mieloide) Os tumores restantes foram tumor ependimário com diferenciação mixopapilar e ependimoblástica, em indiv duos com mutação G870D e um tumor neuroectodérm co primitivo por mutação no J871T.

No grupo de indivíduos com mutação em células germinativas de SETBP1 que desenvolveram cânce a ruptura da interação do gen com a proteína é maio que naqueles que nâo desenvolveram câncer. Analisada a prevalência de malignidade por genótipo em 33 indivíduos com mutação positiva com SGS. Enquanto a incidencia de genese tumoral e de $21,2 \%$ desse grupo, indivíduos com mutação D868 tem um risco maior, estatisticamente significativo de desenvolver tumores que os indivíduos com outras mutações em SETBP1. Inversamente, mutações no resíduo D868 são as mais prevalentes nas mutações somáticas em indivíduos com câncer.

\section{DISCUSSÃO}

Apresentamos 47 indivíduos com SGS causada por mutação em células germinativas em SETBP1, afetando o degron (D868-1871). Todas as mutações foram de novo, embora dois individuos fossem imáos e apresentassem a mesma mutação no resíduo 1871. Esse achado sugere mosaicismo nos pais, porém não havia amostras de sangue dos pais para confirmação.

A primeira publicação sobre SGS, em 1978, foi descrita em dois irmãos, o que, inicialmente, nos fez acreditar tratar-se de doença autossômica recessiva.

SGS é uma doença rara, porém clinicamente bem reconhecida, com fácies típica, alterações neurológicas, varias anomalias congenitas e aumento de risco de malignidade. Apresentam deficiência mental, epilepsia intratavel e cegueira e surdez corticais. Frequentemente apresentam anomallas congênitas de múltiplos órgãos como cardiopatias, malformaçóes renais, genitais e ósseas. Os individuos mais afelados não passam da pri-

Dois indivíduos com mutação atípica no resíduo S867 apresentavam todas as características de SGS, menos a idronefrose (marca registrada da SGS).

Mutações somáticas em SETBP1, observadas em doenças mieloides malignas, mostram um efeito de ganho-de-função na proletna SETBP1, elevando o nive da proteína. Esse efeito de ganho-de-função da proteína tambem ocorre nas mutaçôes das células germinativas, provocando elevação no nível das proteínas.

A mutação D868N de SETBP1é a que mais comumente está associada ao câncer, sendo a que provoca maiores níveis da proteína e maior proliferação celular. Indivíduos com SGS causada por mutação no resíduo D868 têm maior incidência de genese de tumores.

A despeito do aumento de risco de gênese de tumores, a maioria dos indivíduos com SGS não desenvolve câncer. Talvez a explicação para isso seja o óbito precoce nesses pacientes, não dando tempo para vir a desenvolver câncer.

As mutações somáticas têm sido associadas à leucemia mieloide crônica, doença que geralmente ocorre em indivíduos maiores de 60 anos.

Os cânceres embrionários são mais frequentes na SGS. A mutação provoca elevação da proteína SET como resultado da inativação de PP2A.

Existem estudos que tentam desenvolver antagonistas à proteína SET ou tentar ativar PP2A, como o OP449, peptídeo sintético que se liga à proteina SET, ativa PP2A e inibe as celulas de crescimento na leucemia.

Está sendo desenvolvido o componente FTY720 que ativa PP2A.

Esses componentes podem ser úteis no tratamento da SGS, contudo, a janela entre o diagnóstico e o potencial terapêutico da intervenção pode ser mínima. Entretanto, esse tratamento pode prevenir a progressão da neurodegeneração.
REFERÉNCIAS
1. Schinzel A Giedion A A syndromeofseveremdfaceretraction, multipleskullanomalies, clubfeet, andcardiac and renal malformations in sibs. Am J Med Genet. 1978; 1: 361 \pm 375 . https://doi.org/10.1002 ajmg. 1320010402PMID: 665725.

2. Minn D, Christmann D, De Saint-Martin A, Alembik , Eliot M, Mack G, et al. Further clinical and sensoria delineation of Schinzel-Giedion syndrome: Report of two cases. Am J Med Genet. 2002; 109: $211 \pm 217$. https://do 3. Al-Mulg. 10348 PML: 11977181.

3. Al-Mudaffer M, Oley C, Price S, Hayes I, Stewart A, Hall CM, et al. Clinical and radiological findings in Schinze-Giedion syndrome.Eur J Pedlatr. 2008; 167: 1399 1407 https://doi.org/10.1007/s00431-008-0683-4 PMID:

4. Hoischen A, van Bon BWM, Gilissen C, Arts P, van Lier $B$, Steehouwer M, et al. De novo mutations of SETBP1 cause Schinzel-Giedion syndrome. Nat Ge net. 2010; 42: 483 \pm 485 . https://doi.org/10.1038/ng. 58 MID: 20436468

5. Piazza R, Valletta S, Winkelmann N, Redaelli S, Spinelli R, Pirola A, et al. Recurrent SETBP1 mutations in atypical chronic myeloid leukemia. Nat Genet. 2012; 45 +24. https://doi.org/10.1038/ng.2495 PMID: 23222956. 6. Sakaguchi H, Okuno Y, Muramatsu H, Yoshida K Shiraishi Y, Takahashi M, et al. Exome sequencing identifies secondary mutations of SETBP1 and JAK3 in juvenile myelomonocytic leukemia. Nat Genet. 2013; 45: 937 41 . https://doi.org/10.1038/ng.2698 PMID: 23832011.

7. Makishima H, Yoshida K, Nguyen N, Przychodzen B, Sanada M, Okuno Y, et al. Somatic SETBP1 mutations in myeloid malignancies. Nat Genet. 2013; 45: 942 \pm 6 . https:// doi.org/10.1038/ng.2696 PMID: 23832012

8. Hoischen A, Krumm N, Eichler EE. Prioritization of neurodevelopmental disease genes by discovery of new mutations. Nat Neurosci. 2014; 17: 764 \pm 772 . https://doi. org/10.1038/nn.3703 PMID: 24866042.

9. Agha MM, Williams JI, Marrett L, To T, Zipursky A, Dodds $L$. Congenital abnormallies and childhood cancer. Cancer. 2005; 103: 1939 \pm 1948 . https://doi.org/10.1002/ cncr.20985 PMID: 15770693.

10. Bjorge T, Cnattingius S, Lie RT, Tretli S, Engeand A. Cancer Risk in Children with Birth Defects and in Their Families: A Population Based Cohort Study of 5.2 Million Children from Norway and Sweden. Cancer Epidemiol Biomarkers Prev. 2008; 17: 500 \pm 506 . https://doi. org/10.1158/1055-9965.EPI-07- 2630 PMID: 18296646.

1- Serviço de Neurologia do HSI

Endereço para correspondência:

flaca@uol.com.br 